\title{
An Update on Emerging Therapeutic Options for Malignant Pleural Mesothelioma
}

\author{
Alexander Davis ${ }^{1,2}$, Helen $\mathrm{Ke}^{3}$, Steven Kao ${ }^{1,3,4}$, Nick Pavlakis ${ }^{4-6}$ \\ 'Department of Medical Oncology, Chris O'Brien Lifehouse, Camperdown, NSW, Australia; ${ }^{2}$ Department of Medical Oncology, Western Cancer \\ Centre, Dubbo, NSW, Australia; ${ }^{3}$ Asbestos Diseases Research Institute, Rhodes, NSW, Australia; ${ }^{4}$ School of Medicine, University of Sydney, \\ Camperdown, NSW, Australia; ${ }^{5}$ Department of Medical Oncology, Royal North Shore Hospital, St Leonards, NSW, Australia; ${ }^{6}$ Department of Medical \\ Oncology, Genesis Care, St Leonards, NSW, Australia
}

Correspondence: Nick Pavlakis, Department of Medical Oncology, Royal North Shore Hospital, St Leonards, NSW, 2065, Australia, Tel +6I2 9463 II72, Fax +6129463 1092, Email nick.pavlakis@sydney.edu.au

\begin{abstract}
The treatment paradigm for malignant pleural mesothelioma (MPM) has changed little in the last 18 years. Radical intent treatment, consisting of surgical resection, radiotherapy and chemotherapy, has been offered to a highly select few; however, there is little randomised evidence to validate this approach. Prior to 2020 chemotherapy with platinum and an anti-folate was the only intervention with randomised evidence to demonstrate improved overall survival (OS) in MPM. No systemic therapy had been demonstrated to improve OS in the second line setting until 2020. The publication of the Checkmate 743 trial in 2021 demonstrated a survival benefit of combination immunotherapy over standard chemotherapy in newly diagnosed patients with MPM. This finding was shortly followed by the CONFIRM trial which demonstrates a modest but significant survival benefit of second line nivolumab versus placebo in patients having previously received standard chemotherapy. The results of these trials, recent biomarker directed therapy and chemotherapy adjuncts are discussed within this review. The integration of immunotherapy for the few patients in whom radical surgical therapy is intended is currently the subject of clinical trials and offers the prospect of improving outcomes in this rare but devastating disease.
\end{abstract}

Keywords: immune checkpoint inhibitors, immunotherapy, malignant pleural mesothelioma, mesothelioma radiotherapy, mesothelioma surgery, trimodality therapy

\section{Background}

Malignant pleural mesothelioma (MPM) is a rare malignant tumour of the pleural lining of the thoracic cavity. MPM has a poor prognosis with a 5 year overall survival (OS) of $5 \%$ for all stages. ${ }^{1}$ The majority of MPM cases are related to asbestos exposure. ${ }^{2}$ There is a long latency period from exposure to the development of disease with an average period of 40 years. ${ }^{3}$ Patients are typically male and commonly diagnosed in the $8^{\text {th }}$ decade of life. ${ }^{4}$ Despite efforts over the past three decades to reduce or ban the use of asbestos, the peak incidence of asbestos in developed countries has yet to occur. ${ }^{5}$ The incidence of MPM is rising in the developing world and is not expected to peak for another 2 decades. $^{6}$ Hence is it expected to remain a significant and preventable cause of global disease.

MPM is grouped into 3 major histopathological subtypes; epithelioid (69\%), sarcomatoid (19\%) and biphasic (12\%). ${ }^{7}$ The sarcomatoid histological subtype is typically considered to have the worst prognosis and be least sensitive to chemotherapy. ${ }^{7}$ Histology is prognostic in surgically resected patients with the sarcomatoid subtype having a worse OS than patients with epithelioid histology. ${ }^{8}$ Loss of function mutations in BRCA 1 associated protein (BAP1) are a frequent event in MPM with germline mutations denoting an increased risk of MPM. ${ }^{9}$ BAP1 germline alterations can predict for improved overall survival following platinum based chemotherapy. ${ }^{10}$ The pattern of BAP1 immunohistochemical (IHC) staining also varies based on histology and can predict for overall survival with cytoplasmic BAP1 staining being associated with a favourable prognosis in non-epithelioid MPM. ${ }^{11}$

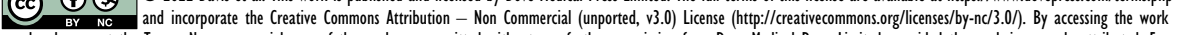
you hereby accept the Terms. Non-commercial uses of the work are permitted without any further permission from Dove Medical Press limited, provided the work is properly attributed. For permission for commercial use of this work, please see paragraphs 4.2 and 5 of our Terms (https://www.dovepress.com/terms.php). 
While surgery, systemic therapy and radiotherapy can be used in the treatment of malignant pleural mesothelioma, systemic therapy is the only treatment which has been demonstrated to improve overall survival in randomised clinical trials. ${ }^{12,14}$ The evidence for local therapies such as surgery and radiotherapy are less robust and will be reviewed in the following section.

\section{Surgery}

The distinct challenges of pleural anatomy make microscopically negative surgical margins for resection of MPM difficult. There is variation in international surgical guidelines as to the ideal surgical approach for MPM patients. ${ }^{15}$ Current NCCN guidelines recommend surgery only when complete macroscopic resection is possible in stage I-IIIA disease, with epithelioid or biphasic histology and in carefully selected patients. Two surgical options are available with the aim of prolonging overall survival: Extrapleural Pneumonectomy (EPP) or extended Pleurectomy/Decortication (eP/D). EPP involves the en bloc resection of the involved lung with visceral pleura, the ipsilateral hemidiaphragm, pericardial and remaining parietal pleura whilst eP/D involves extrapleural dissection to remove the tumour and parietal pleura from the chest wall with resection of the diaphragm and pericardium after which the parietal pleura is resected and the entire visceral pleura dissected to remove visible tumour. ${ }^{16}$ The International Association for the Study of Lung Cancer (IASLC) database of 1494 patients treated with curative intent surgery showed longer OS in stage I disease in patients treated with EPP compared to $\mathrm{P} / \mathrm{D}{ }^{8}$ This retrospective, non-randomized study was collected across 15 centres and four continents and is the largest series to describe surgical outcomes. Since the established sequencing of neoadjuvant chemotherapy, macroscopic complete resection (MCR) surgery and adjuvant radiotherapy by the Memorial Sloan Kettering group, MCR has typically been delivered as part of tri-modality therapy. ${ }^{17}$

The role of EPP was assessed in the multi centre, randomised Mesothelioma and Radical Surgery (MARS) trial in which patients who had completed neoadjuvant chemotherapy were randomised to EPP followed by thoracic radiation or no EPP. ${ }^{18}$ This showed no OS benefit for EPP (median OS 14.4 versus 19.5 months, Hazard Ratio (HR) 1.90, 95\% CI $0.92-3.93$ ) with a hazard ratio suggesting harm with the procedure. However, this trial attracted significant criticism due to its poor accrual and its design. It was underpowered to formally assess the impact on OS and had a comparatively high mortality rate from EPP compared to previously published Phase 2 trials and retrospective series. ${ }^{19}$ No other randomised trials have been subsequently performed looking at EPP.

$\mathrm{eP} / \mathrm{D}$ is an alternative surgical procedure to EPP which also aims to achieve MCR. Meta analyses of phase 2 trials and retrospective data series have identified that patients undergoing $\mathrm{eP} / \mathrm{D}$ have lower perioperative mortality $(2.9 \%$ versus $6.8 \%$ ) and morbidity (27.9\% versus $62.0 \%$ ) than EPP. ${ }^{20,21}$ There was no statistically significant difference in OS in either analysis. While it may be tempting to argue that $\mathrm{eP} / \mathrm{D}$ is a less morbid procedure than EPP, eP/D may be offered to patients with early stage disease whilst EPP may be offered for more advanced (but operable) cases. ${ }^{16}$ In the absence of randomised trials, direct comparisons between the two techniques are difficult. The current MARS2 study is a multicentre, randomised trial looking to compare eP/D versus no surgery in patients following 2 cycle of platinum and pemetrexed chemotherapy. ${ }^{22}$

Thus, there remains no high-level evidence from randomised trials to support routine surgery to achieve MCR in MPM pending the outcome of the MARS2 trial. Moreover, there is ongoing debate in the surgical community as to its overall benefit of MCR intent surgery. In the absence of high quality evidence, surgery remains an option limited to experienced centres for select patients as part of multi-modality treatment. ${ }^{16,23}$

Given the high rate of local recurrence following surgical resection ${ }^{24}$ intraoperative therapy has also been utilised in the surgical management of MPM. A number of single armed studies have demonstrated the safety and feasibility of intracavity cisplatin and gemcitabine following either EPP or P/D. ${ }^{25}$ This has yet to be incorporated into clinical practice guidelines. ${ }^{26}$ Other adjuncts to surgery, such as heated intraoperative povidone-iodine (PVP-I) and photodynamic therapy (PDT), have shown promise in smaller studies yet have not been validated in larger trials and are not included in clinical practice guidelines. ${ }^{27}$ 


\section{Radiotherapy}

Radiotherapy can be used in the treatment of MPM in the peri operative and the palliative setting. ${ }^{28}$ While palliative radiotherapy has often been recommended for the treatment of symptomatic MPM soft tissue or bone metastases, there was limited high quality evidence to support this until the recent single arm, phase 2 SYSTEMS study of 40 patients which found an improvement in pain scores following palliative radiotherapy. ${ }^{29,30}$ The randomized, phase 2 SYSTEMS-2 study is currently underway to assess standard (20Gy/5\#) versus escalated dose $(36 \mathrm{~Gy} / 6 \#)$ palliative radiotherapy on improving pain scores in MPM. ${ }^{31}$

Following a positive randomised controlled trial suggesting benefit for prevention of tract seeding, prophylactic radiotherapy following invasive procedures to the pleural space began to be used in clinical practice. ${ }^{32}$ However, four subsequent randomized controlled trials failed to demonstrate a benefit for prophylactic tract irradiation in reducing the incidence of chest wall recurrence. ${ }^{29-32}$ These discrepant results may reflect the greater efficacy of systemic therapy in contemporary practice. ${ }^{33}$ Based on this collective evidence, prophylactic irradiation of procedural tracts is no longer recommended as standard practice. ${ }^{34}$

In the context of perioperative treatment, radiotherapy can be delivered after neoadjuvant chemotherapy and MCR or alternatively prior to surgery. Hemithoracic radiotherapy can be delivered after EPP with the intent of reducing locoregional recurrence, which can be as high as $29 \%$ at 2 years in some series. ${ }^{35}$ However, there is little randomized data to support this. The phase 2 randomised SAKK 17/04 trial randomised 54 patients who had completed neoadjuvant chemotherapy and EPP to adjuvant hemithoracic radiotherapy or no adjuvant therapy. ${ }^{36}$ When the trial was terminated early due to poor accrual, there was no benefit for hemithoracic radiotherapy compared to no treatment for locoregional relapse free survival (9.4 months versus 7.6 months) or overall survival (19.3 months versus 20.8 months). Criticism of this trial include its design and not being powered to demonstrate an effect on overall survival. Additionally, it included patients with N2 lymph node involvement who may have poor outcomes regardless of adjuvant treatment.

Given the trend away from EPP towards eP/D following the MARS trial there have been concerns regarding the safety of delivering adjuvant radiotherapy with the ipsilateral lung in situ due to the risk of pneumonitis. A single arm, Phase II study to determine the safety of intensity modulated pleural radiation therapy (IMPRINT) following P/D was conducted in 27 patients. ${ }^{37}$ IMPRINT was demonstrated to be safe with no grade 4 or 5 radiation toxicities, but $59 \%$ of patients experienced disease recurrence within the radiation field.

While adjuvant radiotherapy is the dominant paradigm in trimodality therapy for MPM, the role of neoadjuvant radiation was evaluated in the Surgery for Mesothelioma after Radiation Therapy (SMART) clinical trial. ${ }^{38}$ This single arm, phase 2 study delivered $25 \mathrm{~Gy}$ in 5 fractions with a $5 \mathrm{~Gy}$ boost to 96 patients followed by EPP 1 week later. All patients who started treatment completed EPP. Safety analysis revealed $34 \%$ of patients having grade 3 complications within 30 days following pneumonectomy with one patient dying of pneumonia. Adjuvant chemotherapy was considered in $51 \%$ of patients, commenced in $13(14 \%)$ with only 7 patients completing the intended three cycles. Median OS was 24.4 months with $20.1 \%$ of patients having local recurrence. While median OS in patients with epithelioid histology and ypN0 disease was an encouraging 65.9 months, this does come at the cost of significant grade 4 toxicity. The low rate of patients going on to receive chemotherapy also raises questions regarding the optimal sequencing of treatments.

Tri-modality therapy remains the dominant paradigm for patients with MPM being treated with curative intent. However, the evidence base for its individual components such as MCR surgery and hemithoracic radiotherapy remains limited. As the efficacy for systemic therapy increases in the unresectable setting, the benefit for trimodality therapy may be enhanced by the incorporation of these newer agents.

\section{Chemotherapy}

Chemotherapy has been the only modality demonstrated to increase OS for patients with MPM until recently. The landmark Phase 3 clinical trial conducted by Vogelzang et al demonstrated a benefit in median OS (12.1 versus 9.3 months, hazard ratio (HR) $0.77, p=0.02$ ) for the addition of pemetrexed to cisplatin chemotherapy. ${ }^{12}$ There was also a benefit seen with the addition of pemetrexed for median time to progression (5.7 versus 3.9 months, $H R=0.68, p=0.001)$ and response rate $(R R)$ $(41.3 \%$ versus $16.7 \%, \mathrm{p}<0.0001)$. This was associated with increased grade 3 and 4 toxicity such as nausea $(14.6 \%$ versus 
$6.3 \%)$, vomiting ( $13.3 \%$ versus $3.6 \%$ ), diarrhoea ( $4.4 \%$ versus $0 \%$ ), dehydration $(4.0 \%$ versus $0.5 \%)$ and stomatitis $(4.0 \%$ versus $0 \%$ ). There was also an improvement in quality of life for patients receiving combination treatment compared to cisplatin alone. ${ }^{39}$ Based on this study, the combination of cisplatin and pemetrexed received FDA approval for the first line treatment of MPM and has remained the standard of care until recently.

The addition of the vascular endothelial growth factor (VEGF) monoclonal antibody bevacizumab to cisplatin and pemetrexed was evaluated in the phase 3 MAPS study. ${ }^{13}$ This demonstrated a benefit for median OS with the addition of bevacizumab (18.8 versus 16.1 months, HR $0.77, \mathrm{p}=0.0167)$. There was also a benefit seen for median progression free survival (PFS) $(9.2$ versus 7.3 months, HR $0.61, \mathrm{p}<0.0001)$ with the addition of bevacizumab. There was more grade 3 or 4 toxicity with the addition of bevacizumab ( $71 \%$ versus $62 \%$ ) with more patients stopping first line treatment due to treatment toxicity ( $24.3 \%$ versus $6.0 \%, 95 \%$ CI for difference $11.7-24.9, \mathrm{p}<0.0001)$. Grade 3 hypertension $(23 \%$ versus $0 \%)$ and thrombotic events (5.8\% versus $0.9 \%$ ) were particularly elevated with the addition of bevacizumab. Despite the trial showing a statistically significant benefit for OS and being incorporated into clinical practice guidelines, bevacizumab has not received FDA approval for the treatment of MPM. Other strategies to target VEGF have included the use of the angiokinase inhibitor nintedanib in combination with cisplatin and pemetrexed chemotherapy in the Phase III, randomised LUME-Meso trial. ${ }^{40}$ This failed to demonstrate a benefit for median PFS for the addition of nintedanib (6.8 versus 7.0 months, HR $1.0195 \%$ CI $0.79-1.30, \mathrm{p}=0.914)$. Similarly, no benefit was seen for median OS (14.4 versus 16.1 months, HR 1.12; 95\% CI $0.79-1.58, \mathrm{p}=0.538$ ).

Maintenance strategies have been explored to improve clinical outcomes in MPM. The CALB GB 30901 clinical trial assessed 72 MPM patients who had not progressed following platinum and pemetrexed, randomising them to either observation or continuation of pemetrexed. ${ }^{41}$ This did not demonstrate a benefit in PFS (3 versus 3.4 months, HR 0.99; $95 \%$ CI $0.71-1.90, p=0.9733)$. The recent NVALT19, phase 2 open label trial evaluated maintenance gemcitabine chemotherapy versus BSC in patients with no evidence of progression following first-line platinum and pemetrexed containing chemotherapy. ${ }^{42}$ In 130 randomised patients there was a benefit seen for PFS with gemcitabine maintenance (6.2 versus 3.2 months, HR $0.4895 \%$ CI $0.33-0.71, \mathrm{p}=0.0002$ ) compared to BSC. This finding did not translate into a benefit for OS ( 16.4 versus 13.4 months, HR $0.9095 \%$ CI $0.60-1.34, \mathrm{p}=0.60)$. There were more adverse events in the gemcitabine group ( $92 \%$ of patients) compared to BSC (48\% of patients). No quality-of-life data was reported. The authors argued that the high percentage of patients who went on to receive subsequent lines of therapy (83/130 or $63.8 \%$ of patients) may have confounded OS data.

There are limited second line chemotherapy options for patients with MPM. A number of agents have been tried to improve clinical outcomes in patients who have progressed on platinum doublet chemotherapy. Previous phase 3 trials looking at second line pemetrexed added to best supportive care (BSC) for patients treated with previous chemotherapy excluding pemetrexed have failed to demonstrate a benefit for median OS. ${ }^{43}$ Given the majority of patients receive pemetrexed in the first line setting this finding has limited generalizability to current clinical practice. In data recently presented at ASCO 2021 Fennell et al assessed the clinically efficacy of oral vinorelbine and active supportive care (ASC) versus ASC alone in a randomized, phase II trial. ${ }^{44}$ In 154 randomised patients the addition of oral vinorelbine showed a benefit for median PFS (4.2 versus 2.8 months, HR $0.59 ; 95 \%$ CI $0.41-0.85, \mathrm{p}=0.0017$ ). No benefit was seen for median OS (9.3 versus 9.1 months, $\mathrm{HR}=0.79 ; 95 \%$ CI $0.53-1.17, \mathrm{p}=0.24$ ).

The randomised, phase 2 RAMES trial evaluated the benefit for the addition of the VEGFR2 monoclonal antibody ramucirumab to gemcitabine in patients who had progressed following first line treatment for MPM. ${ }^{45}$ Of the 161 patients who received treatment there was a benefit for median OS for the combination therapy group compared to gemcitabine alone (13.8 versus 7.5 months, HR 0.71, $\mathrm{p}=0.057$ ). While this did not meet the criteria for statistical significance, a 6-month benefit for OS in the second line setting could be considered clinically meaningful. More grade 3 and 4 hypertension was observed in the combination therapy arm than for single agent gemcitabine $(6.3 \%$ versus $0 \%, \mathrm{p}=0.022)$. This data has only been presented in abstract form and is yet to enter clinical practice guidelines.

\section{Chemotherapy Adjuncts}

Given MPM is initially a localised disease there has been a desire to find treatments which can be safely directed to the affected pleural cavity. Tumour Treating Fields (TTFields) are a regional treatment for solid tumours which deliver low 
intensity electric fields to the relevant tumour site. ${ }^{46}$ This device acts by disrupting the assembly of the mitotic spindle at metaphase with the field frequency being specific to particular types of cancer cells. ${ }^{47}$ There is randomised phase 3 evidence in glioblastoma multiforme demonstrating that the addition of TTFields to chemotherapy can improve PFS and OS. $^{48}$

The phase 2 STELLAR was a prospective, single arm, multicentre trial looking at the activity of TTFields in combination with platinum and pemetrexed chemotherapy. ${ }^{49}$ For the 80 patients enrolled in the study median OS was 18.2 and median PFS was 7.6 months. There was minimal toxicity associated with the addition of the TTFields device with localised dermatitis occurring in $68 \%$ of patients and was grade 3 in 5\% of patients. Based on this study, TTFields was awarded FDA approval for the treatment of untreated MPM in combination with platinum and pemetrexed chemotherapy. However, given the lack of randomised evidence it has yet to enter clinical practice guidelines.

\section{Immunotherapy}

The chronic inflammatory response to asbestos fibres seen in MPM carcinogenesis leads to an immunosuppressive tumour microenvironment. ${ }^{50}$ Given this, there has been significant interest in exploring the efficacy of immune checkpoint inhibitors (ICI) acting against either the Programmed cell death protein 1(PD-1), Programmed Death Ligand 1 (PD-L1) or Cytotoxic T-Lymphocyte-associated protein 4 (CTLA4) to prompt an anti-tumour immune response. The clinical efficacy of these drugs has been examined in a number of single arm (Table 1) and comparative studies (Table 2).

Many of these studies were conducted in the second line setting in which there is no established treatment for MPM. These non-randomised studies reported median OS ranging from 10-17.3 months with treatment with ICIs such as pembrolizumab or nivolumab. ${ }^{53,54}$ Following these single arm phase 2 studies, ICIs have been evaluated in the second line setting in the randomised PROMISE-Meso and CONFIRM trials.

PROMISE-Meso was a phase 3, multicentre randomised trial of patients with unresectable, pre-treated MPM. ${ }^{59}$ This trial of 144 patients randomised patients on a 1:1 basis to pembrolizumab or institutional choice of chemotherapy (gemcitabine or vinorelbine). Upon progression on chemotherapy patients were allowed to crossover to pembrolizumab. After a median follow up of 11.8 months there was no difference in median OS for pembrolizumab versus chemotherapy (10.7 versus 12.4 months, HR $1.12,95 \%$ CI $0.74-1.69, \mathrm{p}=0.59$ ). There was no benefit in median OS even when adjusting for cross over between the two study groups. Additionally, there was no benefit in median PFS between the

Table I Single Arm Studies of immunotherapy in Mesothelioma

\begin{tabular}{|c|c|c|c|c|c|c|c|}
\hline Study & Drugs \& Schedule & $\mathbf{N}$ & Phase & $\begin{array}{l}\text { Line of } \\
\text { Treatment }\end{array}$ & $\begin{array}{l}\text { Response } \\
\text { Rate }\end{array}$ & PFS (mon) & OS (mon) \\
\hline KEYNOTE028 $8^{51}$ & Pembrolizumab $10 \mathrm{mg} / \mathrm{kg}$ q2 weekly & 25 & $\mathrm{Ib}$ & $2+$ & $20 \%$ & 5.4 & 18 \\
\hline$|R B| 4-|38|^{52}$ & Pembrolizumab $200 \mathrm{mg}$ q3 weekly & 65 & 2 & 2 & $19 \%$ & 4.5 & 11.5 \\
\hline KEYNOTEI $58^{53}$ & Pembrolizumab $200 \mathrm{mg}$ q3 weekly & 118 & 2 & $2+$ & $8 \%$ & 2.1 & 10.0 \\
\hline MERIT $^{54}$ & Nivolumab $240 \mathrm{mg}$ q2 weekly & 34 & 2 & 2 & $29 \%$ & 6.1 & 17.3 \\
\hline INITIATE ${ }^{55}$ & $\begin{array}{l}\text { Nivolumab } 240 \mathrm{mg} \text { q } 2 \text { weekly + ipilimumab } \\
\mathrm{Img} / \mathrm{kg} \text { q } 6 \text { weekly }\end{array}$ & 38 & 2 & $2+$ & $29 \%$ & 6.2 & NR \\
\hline NIBIT-MESO-I ${ }^{56}$ & $\begin{array}{l}\text { Tremelimumab Img/kg + durvalumab } 20 \mathrm{mg} / \mathrm{kg} \\
\text { q4 weekly }\end{array}$ & 40 & 2 & $\mathrm{I}-2$ & $28 \%$ & 5.7 & 16.6 \\
\hline DREAM $^{57}$ & $\begin{array}{l}\text { Cisplatin } 75 \mathrm{mg} / \mathrm{m} 2+\text { pemetrexed } 500 \mathrm{mg} / \mathrm{m} 2+ \\
\text { durvalumab I/ } 25 \mathrm{mg} \text { q3weekly }\end{array}$ & 54 & 2 & I & $48 \%$ & 6.9 & 18.4 \\
\hline $\operatorname{PrE} 0505^{58}$ & $\begin{array}{l}\text { Cisplatin } 75 \mathrm{mg} / \mathrm{m} 2+\text { pemetrexed } 500 \mathrm{mg} / \mathrm{m} 2+ \\
\text { durvalumab } 1 / 20 \mathrm{mg} \text { q3 weekly }\end{array}$ & 55 & 2 & 1 & $56 \%$ & 6.7 & 21.1 \\
\hline
\end{tabular}

Abbreviations: OS, overall survival; PFS, progression free survival; NA, not available; NR, not reached. 
Table 2 Randomised Studies of immunotherapy in Mesothelioma

\begin{tabular}{|c|c|c|c|c|c|c|c|}
\hline Study & Drugs \& Schedule & $\mathbf{N}$ & Phase & $\begin{array}{l}\text { Line of } \\
\text { Treatment }\end{array}$ & $\begin{array}{l}\text { Response } \\
\text { Rate }\end{array}$ & PFS (mon) & OS (mon) \\
\hline PROMISE-Meso ${ }^{59}$ & $\begin{array}{l}\text { Pembrolizumab } 200 \mathrm{mg} \text { q3 weekly versus } \\
\text { chemotherapy }\end{array}$ & 144 & 3 & 2 & $\begin{array}{l}22 \% \text { versus } \\
6 \% *\end{array}$ & $\begin{array}{l}2.5 \text { versus } \\
3.4\end{array}$ & $\begin{array}{l}10.7 \text { versus } \\
12.4\end{array}$ \\
\hline CONFIRM ${ }^{60}$ & Nivolumab $3 \mathrm{mg} / \mathrm{kg}$ q2 weekly versus placebo & 332 & 3 & 2 & NA & $\begin{array}{l}3.0 \text { versus } \\
1.8^{*}\end{array}$ & $\begin{array}{l}9.2 \text { versus } \\
6.6^{*}\end{array}$ \\
\hline MAPS2 ${ }^{61}$ & $\begin{array}{l}\text { Nivolumab } 3 \mathrm{mg} / \mathrm{kg} \text { q2 weekly + Ipilimumab } \\
\mathrm{Img} / \mathrm{kg} \text { q6weekly versus } \\
\text { Nivolumab } 3 \mathrm{mg} / \mathrm{kg} \text { q2weekly }\end{array}$ & 125 & 2 & $2-3$ & $\begin{array}{l}28 \% \text { versus } \\
19 \%\end{array}$ & $\begin{array}{l}5.6 \text { versus } \\
4.0\end{array}$ & $\begin{array}{l}15.9 \text { versus } \\
11.9\end{array}$ \\
\hline CHECKMATE743 $3^{14}$ & $\begin{array}{l}\text { Nivolumab } 3 \mathrm{mg} / \mathrm{kg} \text { q } 2 \text { weekly + ipilimumab } \\
\mathrm{Img} / \mathrm{kg} \text { q } 6 \text { weekly versus platinum + } \\
\text { pemetrexed chemotherapy }\end{array}$ & 605 & 3 & I & $\begin{array}{l}40 \% \text { versus } \\
43 \%\end{array}$ & $\begin{array}{l}6.8 \text { versus } \\
7.2\end{array}$ & $\begin{array}{l}18.1 \text { versus } \\
14 . I^{*}\end{array}$ \\
\hline DETERMINE $^{62}$ & $\begin{array}{l}\text { Tremelimumab } 10 \mathrm{mg} / \mathrm{kg} \text { q3weekly versus } \\
\text { placebo }\end{array}$ & 571 & $2 b$ & $2+$ & $\begin{array}{l}4.5 \% \text { versus } \\
1.1 \%\end{array}$ & $\begin{array}{l}2.8 \text { versus } \\
2.7^{*}\end{array}$ & $\begin{array}{l}7.7 \text { versus } \\
7.3 \text { months }\end{array}$ \\
\hline
\end{tabular}

Note: * denotes statistically significant.

Abbreviations: OS, overall survival; PFS, progression free survival; NA, not available; NR, not reached.

pembrolizumab and chemotherapy groups ( 2.5 versus 3.4 months, HR $1.06,95 \%$ CI $0.73-1.53, \mathrm{p}=0.76$ ). There was an improvement in RR for pembrolizumab compared to chemotherapy (22 versus $6 \%, \mathrm{p}=0.004$ ). Of the 135 patients with available PD-L1 status, 63 (46.7\%) had PD-L1> 1\%. There was no benefit in median OS from pembrolizumab over chemotherapy in this subgroup (13.8 versus 15.3 months, HR $1.0995 \%$ CI $0.57-2.09, \mathrm{p}=0.79$ ). Rates of grade 3 and 4 toxicity were similar between the pembrolizumab (19.4\%) and chemotherapy (25.7\%) arms. Only one case of grade 3 pneumonitis felt to be related to treatment was noted in the pembrolizumab group.

These results are in contrast to the CONFIRM trial, a phase 3, double blind, placebo controlled trial of 12 months of placebo or nivolumab for patients with previously treated mesothelioma ${ }^{60}$ Randomisation to nivolumab was conducted on a 2:1 basis. Cross over was not permitted in the trial, and it is unclear what subsequent therapies were received. Median OS was immature but did show a benefit in favour of nivolumab compared to placebo ( 9.2 versus 6.6 months, HR $0.7295 \%$ CI $0.55-0.94, p=0.018$ ). Investigator assessed median PFS showed a benefit for nivolumab (3 versus 1.8 months, HR 0.61, 95\% CI 0.48-0.77, p < 0.001). There was a benefit seen with nivolumab in median OS for patients with epithelioid histology (9.4 versus 6.6 months, HR $0.7195 \%$ CI $0.53-0.95, \mathrm{p}=0.021$ ) but not in patients with nonepithelioid histology (5.9 versus 6.7 months, HR $0.7995 \%$ CI $0.35-1.79, \mathrm{p}=0.572$ ). Grade 3 and 4 adverse events were $13.1 \%$ in the nivolumab arm and $2.7 \%$ in the placebo arm.

The results of the PROMISE-Meso and CONFIRM studies suggest that ICI such as pembrolizumab and nivolumab has modest but clinically relevant activity in relapsed MPM. ${ }^{63}$ Of note the median OS in the pembrolizumab arm (10.7 months) and nivolumab arm (9.2 months) were similar with marked discrepancy in the chemotherapy (12.4 months) and placebo (6.6 months) comparator arms. There is no evidence to suggest that there is superior activity compared to chemotherapy in the second line setting. The small benefit in OS with second line ICI in MPM has to be weighed against the toxicity and financial cost associated of ICI therapy.

The efficacy of combination ICI with the CTLA monoclonal antibody ipilimumab and nivolumab in untreated MPM was evaluated in the Checkmate 743 study. ${ }^{14}$ This multicentre, randomised, open-label, phase 3 trial compared the combination of ipilimumab and nivolumab to platinum plus pemetrexed chemotherapy in 605 treatment naïve patients with unresectable MPM. At prespecified interim analysis there was an improvement seen for the immunotherapy combination with regards to overall survival (18.1 versus 14.1 months, HR $0.74,96.6 \%$ CI $0.60-0.91, \mathrm{p}=0.002$ ). There was no benefit in median PFS for combination immunotherapy compared to chemotherapy (6.8 versus 7.2 months, HR 1.00, 95\% CI 0.82-1.21) at this interim analysis. Objective response rate (ORR) was reported to be $40 \%$ of the patients in the combination immunotherapy group and $43 \%$ of patients in the chemotherapy group. There was a 
difference seen in median OS benefit from combination immunotherapy in the epithelioid and non-epithelioid subgroups. For patients with epithelioid histology (76\% of patients) there was no statistical benefit in median OS for combination immunotherapy compared to chemotherapy (18.7 versus 16.5 months, HR $0.86,95 \%$ CI $0.69-1.08$ ). In contrast, patients with non-epithelioid histology (24\%) had improved median OS with combination immunotherapy compared to chemotherapy (18.1 versus 8.8 months, HR $0.46,95 \%$ CI $0.31-0.68$ ). Checkmate 743 was not powered to demonstrate a difference between epithelioid and non-epithelioid subgroups. Grade 3 and 4 events were similar in the combination immunotherapy (30\%) and chemotherapy groups (32\%). More patients were likely to suffer an adverse event leading to discontinuation in the combination immunotherapy group (15\%) than the chemotherapy group (7\%). There were 3 treatment related deaths in the combination immunotherapy group and 1 in the chemotherapy group. Treatment with combination immunotherapy also led to a delay in deterioration in quality of life compared to chemotherapy in patient reported outcome data. ${ }^{64}$ Based on this trial ipilimumab and nivolumab have received FDA approval for the first line treatment of unresectable MPM regardless of histology.

The combination of chemotherapy and immunotherapy has been assessed in phase 2 clinical trials. The DREAM study was a single arm, multi-centre phase 2 trial looking at the combination of cisplatin, pemetrexed and the ICI durvalumab for untreated, unresectable MPM. ${ }^{57}$ For the 54 patients enrolled on the study median PFS (by iRECIST) was 7.0 months and median OS was 18.4 months. Grade 3 or 4 immune related adverse event were seen in $15 \%$ of patients with 13\% requiring high dose steroids or immunosuppressive drugs. There were no treatment related deaths. A similar single arm, phase 2 trial (PrE0505) assessed the activity of durvalumab to cisplatin and pemetrexed chemotherapy in 55 patients with treatment naïve, surgically unresectable MPM. ${ }^{58}$ In the 55 patients enrolled there was a median OS of 21.1 months with a 12 -month OS rate of $70 \%$. Median PFS was 6.7 months and $56 \%$ of patients had a partial response as their best response. Adverse events associated with durvalumab were generally reported as being grade 1 or 2 .

The results of the Checkmate 743 study have demonstrated that immunotherapy will play a significant role in the treatment of MPM. The use of combination immunotherapy in patients with non-epithelioid histology has significantly improved survival in a group of patients who typically have a poor prognosis. ${ }^{65}$ However, it is unclear whether combination immunotherapy will improve outcomes for the majority of MPM patients who have epithelioid histology. Several studies have been or are ongoing to evaluate the addition of ICI to standard first line chemotherapy. The phase 3, randomised, DREAM3R study is evaluating the combination of cisplatin, pemetrexed and durvalumab chemotherapy to chemotherapy alone ${ }^{66}$ Similarly, the addition of pembrolizumab to conventional cisplatin and pemetrexed chemotherapy versus chemotherapy alone is being assessed in a large, phase 2/3, randomised trial by the Canadian Cancer Trials Group (NCT02784171). The synergy of ICI such as atezolizumab with agents targeting VEGF such as bevacizumab will be evaluated in the phase 3 BEAT-MESO trial which will assess the addition of atezolizumab to carboplatin, pemetrexed and bevacizumab therapy (NCT03762018). It will be of interest whether the addition of ICI to chemotherapy improves outcomes in epithelioid MPM, which represents the majority of patients with MPM. The results of the PROMISE-Meso and CONFIRM studies suggest there is a role for ICI in the second line treatment of MPM, albeit with modest benefit. However, as the Checkmate 743 has significantly changed the paradigm of initial treatment in MPM, the clinical relevance of these second line studies is restricted to those patients not having received previous immunotherapy as their initial treatment.

\section{Beyond Immune Checkpoint Inhibitors}

Mesothelin (MSLN) is a membrane protein expressed in normal mesothelial cells and in MPM. MSLN is predominantly expressed in the epithelioid histological subtype of MPM. ${ }^{67}$ While MSLN targeted therapy has been evaluated extensively to date in MPM, no agent has been shown to be efficacious in a phase 3 clinical trial. ${ }^{68}$ Using MSLN as a target for Chimeric Antigen Receptor (CAR) T cell therapy has been identified as a potential approach. CAR-T cells are designed $\mathrm{T}$ cell receptor like molecules which possess unique specificity to tumour antigens and can also activate $\mathrm{T}$ cells. A Phase 1 clinical trial in 27 patients with malignant pleural disease (including 25 with MPM) was conducted with CAR $\mathrm{T}$ cell therapy targeting MSLN delivered via intrapleural administration. ${ }^{69}$ All patients had received 1 prior line of therapy. Pembrolizumab was delivered following CAR T cell therapy in 18 patients with median OS of 23.9 months and a 
one-year OS of $83 \%$. Other trials evaluating CAR T cell therapy in MPM using alternative targets are also being explored. $^{70}$

Other approaches to augment the immune response to MPM have also progressed into early phase trials. Approaches using oncoviral and dendritic cell therapy have reached Phase III trials which are currently recruiting. ${ }^{71}$ Given the success of ICI in the treatment of MPM it is anticipated that other approaches co-opting the immune response may offer benefit.

\section{Novel Approaches}

There have been several attempts to develop targeted therapy in MPM. BAP1 is the most commonly altered gene in MPM with $57 \%$ of cases being BAP1 deficient. ${ }^{72}$ BAP1 alterations impede DNA double strand break repair through homologous recombination. ${ }^{73}$ Using poly (ADP-ribose) polymerase (PARP) inhibitors to achieve synthetic lethality has been a successful treatment approach in other malignancies with deficiencies in DNA double strand break repair such as BRCA associated ovarian cancer. ${ }^{74}$ In addition to BAP1 loss it is suggested $38 \%$ of mesothelioma patients may be BRCA deficient. ${ }^{75}$ The phase 2, single arm Mesothelioma Stratified Therapy 1 (MiST1) study assessed the activity of the PARP inhibitor rucaparib in BAP1 or BRCA 1 deficient mesothelioma patients who has progressed on one previous line of therapy. Treatment was continued for 24 weeks. Of the 26 patients in the study $58 \%$ demonstrated disease control at 12 weeks, which was the primary endpoint of the study. After 24 weeks on the study 58\% of patients had progressive disease. Grade 3 or 4 adverse events were demonstrated in $38 \%$ of patients with $12 \%$ suffering anaemia and $12 \%$ having upper respiratory tract infections. While the results of this trial demonstrate some modest activity of PARP inhibitors in BAP1 or BRCA1 deficient MPM, other trials have found minimal benefit for PARP inhibitors in progressive mesothelioma patients with germline BAP1 mutations. ${ }^{76}$

Agents which block the exogenous supply of the amino acid arginine have shown clinical benefit in MPM and in particular tumours which are deficient in enzymes such as argininosuccinate synthetase 1 (ASS1). ${ }^{77}$ A phase 1 trial study assessed the safety and efficacy of the combination of cisplatin and pemetrexed with the arginine degrading pegargiminase in 32 ASS1 deficient patients. ${ }^{78}$ This demonstrated a median PFS of 5.6 months and OS of 10.1 months. Of interest was that more patients were alive with biphasic disease (40\%) at 15 months than with epithelioid disease $(20 \%)$. Additionally, there was a higher survival at 12 months for sarcomatoid patients (30\%) compared to historical controls $(10 \%)$. These encouraging findings have resulted in the randomised, double-blind, phase 2/3 trial ATOMIC-Meso trial which is evaluating the benefit of pegargiminase or placebo to cisplatin and pemetrexed chemotherapy in non-epithelioid MPM.

\section{Future Directions}

The results of Checkmate 743 have demonstrated clear evidence of benefit for combination ICIs in MPM and particularly for the non-epithelioid subgroup which typically has poor outcomes. While this study has solidified the role of immunotherapy in the treatment of MPM, it is not yet clear if this study changes the first line treatment paradigm for all patients (given superiority in outcomes was more modest and not statistically certain for patients with epithelioid histology). However, even if first line combination immunotherapy is only as effective as chemotherapy, the differing toxicity profile and improved quality of life it offers may still be preferable to patients and clinicians. On this basis it has been incorporated into clinical practice guidelines. However, whether combination immunotherapy such as that used in Checkmate 743 is the optimal paradigm for the addition of immunotherapy in MPM may change based on the results of several combination chemoimmunotherapy studies such as DREAM3R, the CCTG study and BEAT-MESO. If chemoimmunotherapy shows a benefit across all histological types and has less immune related toxicity this approach could become a preferred treatment combination in some patients. In the second line setting the CONFIRM trial demonstrated modest benefit with single agent nivolumab. The generalizability and clinical impact of this will become less relevant as more patients receive immunotherapy in the first line setting.

The improvement in systemic therapy for MPM may impact on the other treatment modalities used for MPM. Patients with sarcomatoid MPM have not been viewed favourably as candidates for trimodality therapy due to inferior clinical outcomes. The use of neoadjuvant ICI has been evaluated in a number of Phase I \& II trials which are yet to report their 


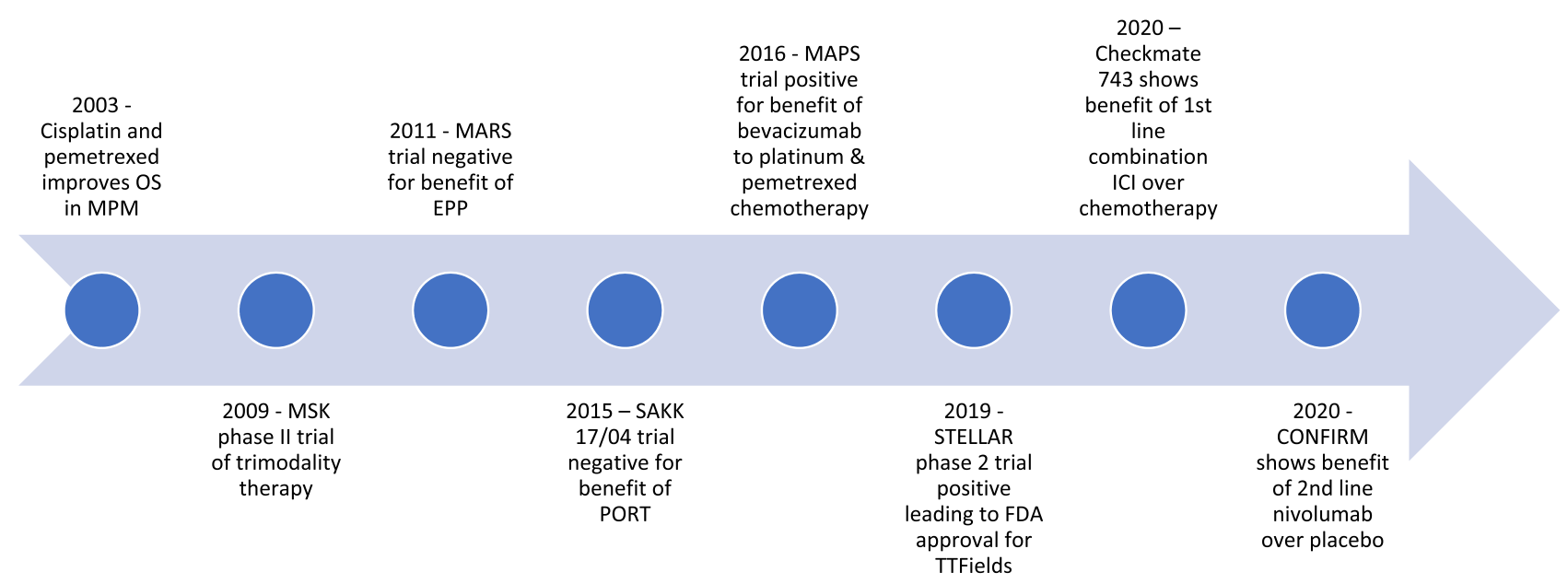

Figure I Timeline of key phase positive and negative 2 and 3 trials in MPM for surgery, radiotherapy and systemic therapy.

Abbreviations: OS, overall survival; EPP, extra pleural pneumonectomy; TTFields, tumour treating fields; ICI, immune checkpoint inhibitor; PORT, post-operative radiotherapy.

findings. ${ }^{79}$ Should this approach prove successful this may increase the number of patients eligible for trimodality therapy in specialised centres.

The initial results from the MiST1 study offer the promise of personalised medicine in MPM. This is part of a multiarm, phase 2 clinical trial exploring biomarker driver therapy. ${ }^{80}$ Other cohorts within the study include the CDK4/6 inhibitor abemaciclib for p16ink4a deficient tumours, the bevacizumab and atezolizumab combination for PDL1 positive tumours and pembrolizumab and the AXL kinase inhibitor bemcentinib for patients without biomarkers. This approach may lead to better outcomes for selected patients with MPM.

Figure 1 has demonstrated the timeline for new evidence-based practice in MPM. Further trials looking at other means of improving the immune response in MPM are currently underway with the hope of further improving outcomes. ${ }^{81}$

\section{Conclusion}

After more than a decade of minimal improvement in the first line treatment of advanced MPM, the Checkmate 743 clinical trial cemented the role of combination immunotherapy in the treatment of this disease. However, three large randomised controlled trials are ongoing to evaluate the benefit of immunotherapy combined with standard chemotherapy. Whether combination immunotherapy or chemoimmunotherapy becomes the dominant paradigm for all patients remains to be seen. Clinical studies are also currently underway evaluating immunotherapy in the minority of patients with MPM who may be eligible for trimodality treatments. Ongoing research also continues into targeted approaches and novel immune therapies. The way forward in improving overall survival and quality of life for patients with advanced MPM will require choosing the appropriate treatment paradigm for patients based on their tumour characteristics.

\section{Disclosure}

HK is the scholarship recipient from Asbestos Disease Foundation of Australia. SK reports honoraria to Institution from Astra Zeneca, Roche, Boehringer, Pfizer, BMS, MSD and Takeda; research funding to institution from Astra Zeneca; travel \& accommodation from BMS, Roche \& Boehringer. NP serves in advisory boards for Boehringer Ingelheim, MSD, Merck, BMS, Astra Zeneca, Takeda, Pfizer, and Roche; reports speaking honoraria from Boehringer Ingelheim, Bayer, Novartis, Pfizer, Roche, Takeda, and Ipsen; research funding to institution from Bayer, Pfizer, and Roche. The authors report no other conflicts of interest in this work. 


\section{References}

1. Shavelle R, Vavra-Musser K, Lee J, Brooks J. Life expectancy in pleural and peritoneal mesothelioma. Lung Cancer Int. 2017;2017:1-8. doi:10.1155/2017/2782590

2. Spirtas R, Heineman EF, Bernstein L, et al. Malignant mesothelioma: attributable risk of asbestos exposure. Occup Environ Med. 1994;51(12):804811.

3. Alpert N, van Gerwen M, Taioli E. Epidemiology of mesothelioma in the 21 st century in Europe and the United States, 40 years after restricted/ banned asbestos use. Transl Lung Cancer Res. 2020;9(Suppl 1):S28-S38.

4. Saddoughi SA, Abdelsattar ZM, Blackmon SH. National trends in the epidemiology of malignant pleural mesothelioma: a national cancer data base study. Ann Thorac Surg. 2018;105(2):432-437.

5. Robinson BM. Malignant pleural mesothelioma: an epidemiological perspective. Ann Cardiothorac Surg. 2012;1(4):491-496.

6. Kwak KM, Paek D, Hwang S, Ju YS. Estimated future incidence of malignant mesothelioma in South Korea: projection from 2014 to 2033. Pershouse MA, ed. PLoS One. 2017;12(8):e0183404.

7. Meyerhoff RR, Yang CFJ, Speicher PJ, et al. Impact of mesothelioma histologic subtype on outcomes in the Surveillance, Epidemiology, and End Results database. J Surg Res. 2015;196(1):23-32.

8. Rusch VW, Giroux D, Kennedy C, et al. Initial analysis of the international association for the study of lung cancer mesothelioma database. $J$ Thorac Oncol. 2012;7(11):1631-1639.

9. Cigognetti M, Lonardi S, Fisogni S, et al. BAP1 (BRCA1-associated protein 1) is a highly specific marker for differentiating mesothelioma from reactive mesothelial proliferations. Mod Pathol. 2015;28(8):1043-1057.

10. Hassan R, Morrow B, Thomas A, et al. Inherited predisposition to malignant mesothelioma and overall survival following platinum chemotherapy. Proc Natl Acad Sci. 2019;116(18):9008-9013.

11. De Rienzo A, Chirieac LR, Hung YP, et al. Large-scale analysis of BAP1 expression reveals novel associations with clinical and molecular features of malignant pleural mesothelioma. J Pathol. 2021;253(1):68-79.

12. Vogelzang NJ, Rusthoven JJ, Symanowski J, et al. Phase III study of pemetrexed in combination with cisplatin versus cisplatin alone in patients with malignant pleural mesothelioma. J Clin Oncol. 2003;21(14):2636-2644.

13. Zalcman G, Mazieres J, Margery J, et al. Bevacizumab for newly diagnosed pleural mesothelioma in the Mesothelioma Avastin Cisplatin Pemetrexed Study (MAPS): a randomised, controlled, open-label, phase 3 trial. The Lancet. 2016;387(10026):1405-1414.

14. Baas P, Scherpereel A, Nowak AK, et al. First-line nivolumab plus ipilimumab in unresectable malignant pleural mesothelioma (CheckMate 743): a multicentre, randomised, open-label, phase 3 trial. The Lancet. 2021;397(10272):375-386.

15. Ricciardi S, Cardillo G, Zirafa CC, et al. Surgery for malignant pleural mesothelioma: an international guidelines review. J Thorac Dis. 2018;10 (Suppl 2):S285-S292.

16. Bueno R, Opitz I. Surgery in Malignant Pleural Mesothelioma. J Thorac Oncol. 2018;13(11):1638-1654.

17. Krug LM, Pass HI, Rusch VW, et al. Multicenter phase II trial of neoadjuvant pemetrexed plus cisplatin followed by extrapleural pneumonectomy and radiation for malignant pleural mesothelioma. J Clin Oncol off J Am Soc Clin Oncol. 2009;27(18):3007-3013.

18. Treasure T, Lang-Lazdunski L, Waller D, et al. Extra-pleural pneumonectomy versus no extra-pleural pneumonectomy for patients with malignant pleural mesothelioma: clinical outcomes of the Mesothelioma and Radical Surgery (Mars) randomised feasibility study. Lancet Oncol. 2011;12 (8):763-772.

19. Weder W, Stahel RA, Baas P, et al. The Mars feasibility trial: conclusions not supported by data. Lancet Oncol. 2011;12(12):1093-1094;author reply 1094-1095.

20. Cao C, Tian D, Park J, Allan J, Pataky KA, Yan TD. A systematic review and meta-analysis of surgical treatments for malignant pleural mesothelioma. Lung Cancer. 2014;83(2):240-245.

21. Taioli E, Wolf AS, Flores RM. Meta-analysis of survival after pleurectomy decortication versus extrapleural pneumonectomy in mesothelioma. Ann Thorac Surg. 2015;99(2):472-480.

22. Lim E, Darlison L, Edwards J, et al. Mesothelioma and Radical Surgery 2 (Mars 2): protocol for a multicentre randomised trial comparing (extended) pleurectomy decortication versus no (extended) pleurectomy decortication for patients with malignant pleural mesothelioma. BMJ Open. 2020;10(9):e038892.

23. Kantor T, Wakeam E. Landmark trials in the surgical management of mesothelioma. Ann Surg Oncol. 2021;28(4):2037-2047.

24. Baldini MDEH, Recht MDA, Strauss MDGM, et al. Patterns of failure after trimodality therapy for malignant pleural mesothelioma. Ann Thorac Surg. 1997;63(2):334-338.

25. Burt BM, Richards WG, Lee HS, et al. A Phase I trial of surgical resection and intraoperative hyperthermic cisplatin and gemcitabine for pleural mesothelioma. J Thorac Oncol. 2018;13(9):1400-1409.

26. Migliore M, Ried M, Molins L, et al. Hyperthermic intrathoracic chemotherapy (HITHOC) should be included in the guidelines for malignant pleural mesothelioma. Ann Transl Med. 2021;9(11):960.

27. Chan WH, Sugarbaker DJ, Burt BM. Intraoperative adjuncts for malignant pleural mesothelioma. Transl Lung Cancer Res. 2017;6(3):285-294.

28. Gomez DR, Rimner A, Simone CB, et al. The Use of Radiation Therapy for the Treatment of Malignant Pleural Mesothelioma: expert Opinion from the National Cancer Institute Thoracic Malignancy Steering Committee, International Association for the Study of Lung Cancer, and Mesothelioma Applied Research Foundation. J Thorac Oncol. 2019;14(7):1172-1183.

29. MacLeod N, Price A, O’Rourke N, Fallon M, Laird B. Radiotherapy for the treatment of pain in malignant pleural mesothelioma: a systematic review. Lung Cancer. 2014;83(2):133-138.

30. MacLeod N, Chalmers A, O’Rourke N, et al. Is radiotherapy useful for treating pain in mesothelioma?: a Phase II Trial. J Thorac Oncol off Publ Int Assoc Study Lung Cancer. 2015;10(6):944-950.

31. Ashton M, O'Rourke N, Macleod N, et al. SYSTEMS-2: a randomised phase II study of radiotherapy dose escalation for pain control in malignant pleural mesothelioma. Clin Transl Radiat Oncol. 2018;8:45-49.

32. Boutin C, Rey F, Viallat JR. Prevention of malignant seeding after invasive diagnostic procedures in patients with pleural mesothelioma. Chest. 1995;108(3):754-758.

33. Hanna GG, John T, Ball DL. Controversies in the role of radiotherapy in pleural mesothelioma. Transl Lung Cancer Res. 2021;10(4):2079-2087. 
34. Kindler HL, Ismaila N, Armato SG, et al. Treatment of malignant pleural mesothelioma: American society of clinical oncology clinical practice guideline. J Clin Oncol off J Am Soc Clin Oncol. 2018;36(13):1343-1373.

35. Gomez DR, Hong DS, Allen PK, et al. Patterns of failure, toxicity, and survival after extrapleural pneumonectomy and hemithoracic intensitymodulated radiation therapy for malignant pleural mesothelioma. J Thorac Oncol off Publ Int Assoc Study Lung Cancer. 2013;8(2):238-245.

36. Stahel RA, Riesterer O, Xyrafas A, et al. Neoadjuvant chemotherapy and extrapleural pneumonectomy of malignant pleural mesothelioma with or without hemithoracic radiotherapy (SAKK 17/04): a randomised, international, multicentre phase 2 trial. Lancet Oncol. 2015;16(16):1651-1658.

37. Rimner A, Zauderer MG, Gomez DR, et al. Phase II Study of Hemithoracic Intensity-Modulated Pleural Radiation Therapy (IMPRINT) as part of lung-sparing multimodality therapy in patients with malignant pleural mesothelioma. J Clin Oncol off J Am Soc Clin Oncol. 2016;34(23):27612768.

38. Cho BCJ, Donahoe L, Bradbury PA, et al. Surgery for malignant pleural mesothelioma after radiotherapy (SMART): final results from a singlecentre, phase 2 trial. Lancet Oncol. 2021;22(2):190-197.

39. Boyer MJ, Jassem J, Liepa AM, et al. O-56 Symptom and quality of life advantages for pemetrexed + cisplatin versus cisplatin in treatment of malignant pleural mesothelioma. Lung Cancer. 2003;41:S19.

40. Scagliotti GV, Gaafar R, Nowak AK, et al. Nintedanib in combination with pemetrexed and cisplatin for chemotherapy-naive patients with advanced malignant pleural mesothelioma (LUME-Meso): a double-blind, randomised, placebo-controlled phase 3 trial. Lancet Respir Med. 2019;7 (7):569-580.

41. Dudek AZ, Wang X, Gu L, et al. Randomized study of maintenance pemetrexed versus observation for treatment of malignant pleural mesothelioma: CALGB 30901. Clin Lung Cancer. 2020;21(6):553-561.e1.

42. de Gooijer CJ, van der Noort V, Stigt JA, et al. Switch-maintenance gemcitabine after first-line chemotherapy in patients with malignant mesothelioma (NVALT19): an investigator-initiated, randomised, open-label, phase 2 trial. Lancet Respir Med. 2021;9(6):585-592.

43. Jassem J, Ramlau R, Santoro A, et al. Phase III trial of pemetrexed plus best supportive care compared with best supportive care in previously treated patients with advanced malignant pleural mesothelioma. J Clin Oncol. 2008;26(10):1698-1704.

44. Fennell DA, Casbard AC, Porter C, et al. A randomized phase II trial of oral vinorelbine as second-line therapy for patients with malignant pleural mesothelioma. J Clin Oncol. 2021;39(15_suppl):8507.

45. Pagano M, Ceresoli GL, Zucali PA, et al. RAMES trial: a multicentre, double-blind, randomized, phase II study on gemcitabine plus ramucirumab versus gemcitabine alone as second-line treatment for advanced malignant pleural mesothelioma (MPM). Ann Oncol. 1900P;2020(31):S1078.

46. Mun EJ, Babiker HM, Weinberg U, Kirson ED, Von Hoff DD. Tumor-treating fields: a fourth modality in cancer treatment. Clin Cancer Res off J Am Assoc Cancer Res. 2018;24(2):266-275.

47. Giladi M, Schneiderman RS, Voloshin T, et al. Mitotic spindle disruption by alternating electric fields leads to improper chromosome segregation and mitotic catastrophe in cancer cells. Sci Rep. 2016;5(1):18046.

48. Stupp R, Taillibert S, Kanner A, et al. Effect of tumor-treating fields plus maintenance temozolomide vs maintenance temozolomide alone on survival in patients with glioblastoma: a randomized clinical trial. JAMA. 2017;318(23):2306-2316.

49. Ceresoli GL, Aerts JG, Dziadziuszko R, et al. Tumour Treating Fields in combination with pemetrexed and cisplatin or carboplatin as first-line treatment for unresectable malignant pleural mesothelioma (STELLAR): a multicentre, single-arm phase 2 trial. Lancet Oncol. 2019;20(12):17021709.

50. Yap TA, Aerts JG, Popat S, Fennell DA. Novel insights into mesothelioma biology and implications for therapy. Nat Rev Cancer. 2017;17(8):475488.

51. Alley EW, Lopez J, Santoro A, et al. Clinical safety and activity of pembrolizumab in patients with malignant pleural mesothelioma (KEYNOTE028): preliminary results from a non-randomised, open-label, phase 1b trial. Lancet Oncol. 2017;18(5):623-630.

52. Desai A, Karrison T, Rose B, et al. OA08.03 Phase II Trial of Pembrolizumab (NCT02399371) in previously-treated malignant Mesothelioma (MM): final analysis. $J$ Thorac Oncol. 2018;13(10):S339.

53. Yap TA, Nakagawa K, Fujimoto N, et al. Efficacy and safety of pembrolizumab in patients with advanced mesothelioma in the open-label, singlearm, phase 2 KEYNOTE-158 study. Lancet Respir Med. 2021;2021:S2213260020305154.

54. Okada M, Kijima T, Aoe K, et al. Clinical Efficacy and Safety of Nivolumab: results of a Multicenter, Open-label, Single-arm, Japanese Phase II study in Malignant Pleural Mesothelioma (MERIT). Clin Cancer Res. 2019;25(18):5485-5492.

55. Disselhorst MJ, Quispel-Janssen J, Lalezari F, et al. Ipilimumab and nivolumab in the treatment of recurrent malignant pleural mesothelioma (INITIATE): results of a prospective, single-arm, phase 2 trial. Lancet Respir Med. 2019;7(3):260-270.

56. Calabrò L, Morra A, Giannarelli D, et al. Tremelimumab combined with durvalumab in patients with mesothelioma (NIBIT-MESO-1): an openlabel, non-randomised, phase 2 study. Lancet Respir Med. 2018;6(6):451-460.

57. Nowak AK, Lesterhuis WJ, Kok PS, et al. Durvalumab with first-line chemotherapy in previously untreated malignant pleural mesothelioma (DREAM): a multicentre, single-arm, phase 2 trial with a safety run-in. Lancet Oncol. 2020;21(9):1213-1223.

58. Forde PM, Sun Z, Anagnostou V, et al. PrE0505: phase II multicenter study of anti-PD-L1, durvalumab, in combination with cisplatin and pemetrexed for the first-line treatment of unresectable malignant pleural mesothelioma (MPM) - a PrECOG LLC study. J Clin Oncol. 2020;38 (15_suppl):9003.

59. Popat S, Curioni-Fontecedro A, Dafni U, et al. A multicentre randomised phase III trial comparing pembrolizumab versus single-agent chemotherapy for advanced pre-treated malignant pleural mesothelioma: the European Thoracic Oncology Platform (ETOP 9-15) PROMISEmeso trial. Ann Oncol. 2020;31(12):1734-1745.

60. Fennell D, Ottensmeier C, Califano R, et al. PS01.11 nivolumab versus placebo in relapsed malignant mesothelioma: the CONFIRM Phase 3 Trial. $J$ Thorac Oncol. 2021;16(3):S62.

61. Scherpereel A, Mazieres J, Greillier L, et al. Nivolumab or nivolumab plus ipilimumab in patients with relapsed malignant pleural mesothelioma (IFCT-1501 MAPS2): a multicentre, open-label, randomised, non-comparative, phase 2 trial. Lancet Oncol. 2019;20(2):239-253.

62. Maio M, Scherpereel A, Calabrò L, et al. Tremelimumab as second-line or third-line treatment in relapsed malignant mesothelioma (DETERMINE): a multicentre, international, randomised, double-blind, placebo-controlled phase 2b trial. Lancet Oncol. 2017;18(9):1261-1273.

63. Cui W, Popat S. Immune checkpoint inhibition for unresectable malignant pleural mesothelioma. Drugs. 2021;81(9):971-984. 
64. Scherpereel A, Antonia S, Bautista Y, et al. LBA1 First-line nivolumab (NIVO) plus ipilimumab (IPI) versus chemotherapy (chemo) for the treatment of unresectable malignant pleural mesothelioma (MPM): patient-reported outcomes (PROs) from CheckMate 743. Ann Oncol. 2020;31: S1441.

65. Van Gerwen M, Alpert N, Wolf A, et al. Prognostic factors of survival in patients with malignant pleural mesothelioma: an analysis of the National Cancer Database. Carcinogenesis. 2019;40(4):529-536.

66. Forde PM, Nowak AK, Kok PS, et al. DREAM3R: durvalumab with chemotherapy as first-line treatment in advanced pleural mesothelioma-a phase 3 randomized trial. $J$ Clin Oncol. 2021;39(15_suppl):TPS8586-TPS8586.

67. Ordóñez NG. Value of mesothelin immunostaining in the diagnosis of mesothelioma. Mod Pathol. 2003;16(3):192-197.

68. Lv J, Li P. Mesothelin as a biomarker for targeted therapy. Biomark Res. 2019;7(1):18.

69. Adusumilli PS, Zauderer MG, Riviere I, et al. A phase I trial of regional mesothelin-targeted CAR T-cell therapy in patients with malignant pleural disease, in combination with the anti-PD-1 agent pembrolizumab. Cancer Discov. 2021;11:2768.

70. Curioni A, Britschgi C, Hiltbrunner S, et al. A phase I clinical trial of malignant pleural mesothelioma treated with locally delivered autologous anti-FAP-targeted CAR T-cells. Ann Oncol. 2019;30:v501.

71. Davis AP, Kao SC, Clarke SJ, Boyer M, Pavlakis N. Emerging biological therapies for the treatment of malignant pleural mesothelioma. Expert Opin Emerg Drugs. 2021;26(2):179-192.

72. Hmeljak J, Sanchez-Vega F, Hoadley KA, et al. Integrative molecular characterization of malignant pleural mesothelioma. Cancer Discov. 2018;8 (12):1548-1565.

73. Ismail IH, Davidson R, Gagné JP, Xu ZZ, Poirier GG, Hendzel MJ. Germline mutations in BAP1 impair its function in DNA double-strand break repair. Cancer Res. 2014;74(16):4282-4294.

74. Moore K, Colombo N, Scambia G, et al. Maintenance olaparib in patients with newly diagnosed advanced ovarian cancer. N Engl J Med. $2018 ; 379$ (26):2495-2505.

75. Busacca S, Sheaff M, Arthur K, et al. BRCA1 is an essential mediator of vinorelbine-induced apoptosis in mesothelioma. $J$ Pathol. 2012;227 (2):200-208

76. Hassan R, Mian I, Wagner C, et al. Phase II study of olaparib in malignant mesothelioma (MM) to correlate efficacy with germline and somatic mutations in DNA repair genes. J Clin Oncol. 2020;38(15_suppl):9054.

77. Szlosarek PW, Steele JP, Nolan L, et al. Arginine deprivation with pegylated arginine deiminase in patients with argininosuccinate synthetase 1deficient malignant pleural mesothelioma: a randomized clinical trial. JAMA Oncol. 2017;3(1):58.

78. Szlosarek PW, Phillips MM, Pavlyk I, et al. Expansion Phase 1 study of pegargiminase plus pemetrexed and cisplatin in patients with argininosuccinate synthetase 1-deficient mesothelioma: safety, efficacy, and resistance mechanisms. JTO Clin Res Rep. 2020;1(4):100093.

79. Gray SG. Emerging avenues in immunotherapy for the management of malignant pleural mesothelioma. BMC Pulm Med. 2021;21(1):148

80. Fennell D, Hudka M, Darlison L, et al. P2.06-02 Mesothelioma Stratified Therapy (MiST): a Phase IIA umbrella trial for accelerating the development of precision medicines. J Thorac Oncol. 2019;14(10):S755-S756.

81. de Gooijer CJ, Borm FJ, Scherpereel A, Baas P. Immunotherapy in malignant pleural mesothelioma. Front Oncol. 2020;10:187.

Lung Cancer: Targets and Therapy

Dovepress

\section{Publish your work in this journal}

Lung Cancer: Targets and Therapy is an international, peer-reviewed, open access journal focusing on lung cancer research, identification of therapeutic targets and the optimal use of preventative and integrated treatment interventions to achieve improved outcomes, enhanced survival and quality of life for the cancer patient. Specific topics covered in the journal include: Epidemiology, detection and screening; Cellular research and biomarkers; Identification of biotargets and agents with novel mechanisms of action; Optimal clinical use of existing anticancer agents, including combination therapies; Radiation and surgery; Palliative care; Patient adherence, quality of life, satisfaction; Health economic evaluations.

Submit your manuscript here: http://www.dovepress.com/lung-cancer-targets-therapy-journal 